Review

\title{
Geometry of Multiscale Nonequilibrium Thermodynamics
}

\section{Miroslav Grmela}

École Polytechnique de Montréal, C.P. 6079, succursale Centre-ville, Montréal H3C 3A7, QC, Canada; E-Mail: miroslav.grmela@polymtl.ca

Academic Editor: George Ruppeiner

Received: 2 June 2015 / Accepted: 20 August 2015 / Published: 25 August 2015

\begin{abstract}
The time evolution of macroscopic systems can be experimentally observed and mathematically described on many different levels of description. It has been conjectured that the governing equations on all levels are particular realizations of a single abstract equation. We support this conjecture by interpreting the abstract equation as a geometrical formulation of general nonequilibrium thermodynamics.
\end{abstract}

Keywords: equilibrium thermodynamics; nonequilibrium thermodynamics; contact geometry; GENERIC

\section{Introduction}

Euler-Navier-Stokes-Fourier fluid mechanics and Boltzmann kinetic theory are two examples of mesoscopic dynamical theories. Their governing equations, as well as governing equations of many other well-established (i.e., extensively tested with the results of experimental observations) mesoscopic dynamical theories, can be formally written as:

$$
\dot{x}=L E_{x}+\left[\chi \Xi_{x^{*}}\right]_{x^{*}=S_{x}}
$$

where $x$ stands for the state variable (hydrodynamic fields in the Euler-Navier-Stokes-Fourier fluid mechanics and one particle distribution function in the Boltzmann kinetic theory), $\dot{x}$ denotes the time derivative of $x, E(x)$ is the energy and $S(x)$ is the entropy. By $E_{x}$, we denote the derivative of $E$ with respect to $x$; similarly, $S_{x}$ its derivative of $S$ with respect to $x$; and $\Xi_{x^{*}}$ is the derivative of $\Xi\left(x, x^{*}\right)$, called a dissipation potential, with respect to $x^{*}$. By $L$, we denote an operator transforming the covector $E_{x}$ into a vector, and $\chi \in \mathbb{R}^{+}$is a parameter. The operator $L$ is conveniently defined (see more in Section 3.1) by the Poisson bracket $\{A, B\}=<A_{x}, L B_{x}>$, where $A$ and $B$ are real valued functions of 
$x$, and $<,>$ is a scalar product in the space whose elements are the state variables $x$. The first term on the right-hand side of (1) represents the Euler part, and the second term the Navier-Stokes-Fourier part in fluid mechanics equations. In the Boltzmann kinetic equation, the first term represents the free flow and the second term binary collisions.

A time evolution Equation (1) in which all of the quantities appearing in it acquire a concrete identity are called a particular realization of (1). For example, the Boltzmann kinetic equation and the Euler-Navier-Stokes-Fourier fluid mechanics equations are two particular realizations of (1) (see Section 3). We shall present general definitions of the quantities appearing in (1), as well as the specific form that they take in some selected particular realizations in Section 3.

In order to be able to see (1) as a concrete time evolution equation already at this point, we may ask the question of what is its simplest non-trivial particular realization. The following time evolution equation is an example of such a realization:

$$
\frac{d}{d t}\left(\begin{array}{c}
p \\
q \\
e
\end{array}\right)=\left(\begin{array}{ccc}
0 & -1 & \frac{S_{q}}{S_{e}} \\
1 & 0 & -\frac{S_{p}}{S_{e}} \\
-\frac{S_{q}}{S_{e}} & \frac{S_{p}}{S_{e}} & 0
\end{array}\right)\left(\begin{array}{l}
0 \\
0 \\
1
\end{array}\right)+\left(\begin{array}{c}
{\left[\Xi_{p^{*}}\right]_{p^{*}=S_{p}}} \\
0 \\
0
\end{array}\right)
$$

In this equation, $x=(p, q, e) \in \mathbb{R}^{3}$. The energy $E(x)=e$ (so that $E_{x}=(0,0,1)^{T}$ ); the entropy $S: \mathbb{R}^{3} \rightarrow \mathbb{R}$ is a sufficiently regular concave function satisfying $S_{e}>0,\left(p^{*}, q^{*}, e^{*}\right)=x^{*}=S_{x}=$ $\left(S_{p}, S_{q}, S_{e}\right)$; and $\Xi: \mathbb{R} \rightarrow \mathbb{R} ;\left(p^{*}\right) \mapsto \Xi\left(p^{*}\right)$ is a sufficiently regular function satisfying the following three properties: $\Xi(0)=0$; $\Xi$ reaches its minimum at $p^{*}=0$; and $\Xi$ is a convex function in a neighbourhood of $p^{*}=0$. The entropy makes its appearance in the mechanical part of the time evolution (governed by the first term on the right-hand side of (2)) in order to guarantee that entropy remains unchanged in such a time evolution (see the details in Section 3.1 below). We shall use this simple representation of (1) to illustrate the abstract mathematical concepts introduced in the following sections.

In Section 2, we recall the history of (1), its name general equation for non-equilibrium reversible-irreversible coupling (GENERIC) and collect physical arguments supporting its pertinence. In Section 3, we provide a detailed mathematical formulation of (1) and derive some qualitative properties of its solutions. The main part of this paper is Section 4, where we explore the thermodynamic and geometric content of (1).

\section{Physics of GENERIC}

Before entering the details of the mathematical structure of Equation (1), we recapitulate its physical basis. There are essentially three routes leading to (1).

\subsection{The First Route to GENERIC: Common Structure}

On this route, the structure of (1) emerges as a common mathematical structure of well-established dynamical theories. The first step was made by Clebsch [1], who realized that the particle dynamics and the Euler fluid mechanics share the structure of Hamiltonian dynamics (that is represented in (1) by the first term on its right-hand side). The investigation initiated by Clebsch then continued in particular in the works of Arnold [2], Marsden and Weinstein [3]. Independently, Landau and Ginzburg [4] and Cahn and 
Hilliard [5] have recognized a common structure of gradient dynamics in the part of the time evolution that is represented in (1) by the second term on its right-hand side (i.e., the Navier-Stokes-Fourier part of the fluid mechanics equations and the Boltzmann collision term in the Boltzmann kinetic equation). Time evolution equations involving both the Hamiltonian and the gradient part had appeared first in [6], in [7] (that was presented at the AMS-IMS-SIAM Joint Summer Research Conference in the Mathematical Sciences on Fluids and Plasmas:Geometry and Dynamics, held at the University of Colorado, Boulder, CO, USA, 17-23 July 1983) and in [8-11]. In [12,13], the abstract Equation (1) has been called GENERIC.

Of course, "a common mathematical structure" is not unique. It depends on the pool of dynamical theories in which it is searched and also on the focus of attention. For example, a different pool of dynamical theories and a different focus has led in [14] to a different mathematical structure that is known as rational thermodynamics. To support (1), we emphasize that (1) has been extracted from a very large pool of dynamical theories (much larger than the pool from which the mathematical structure of rational thermodynamics has been extracted) ranging from most microscopic (as for instance, the particle dynamics) to most macroscopic (as for instance, the level of the classical nonequilibrium thermodynamics and the level on which chemical kinetics is formulated). In addition, there are still two independent routes leading to the same structure of (1). These two routes are described below.

\subsection{The Second Route to GENERIC: Agreement with the Results of Observations}

Mathematical models of macroscopic systems are formulated in order to provide an understanding of their observed behaviour. The pertinence and correctness of the mathematical formulation is validated by making a comparison of theoretical predictions with the results of experimental observations. There are two types of such observations. First, there are observations of specific responses to specific externally-controlled stimuli (we shall call them quantitative observations), and second, there are observations of a more qualitative nature. As for the latter, we recall, for example, that most macroscopic systems, if left for a sufficiently long time without external influences, reach states at which their behaviour is found to be well described by the classical equilibrium thermodynamics. As a part of the validation of the mathematical model, this (or a similar) observation has to be found in agreement with theoretical predictions. While the validation with quantitative observation requires detailed knowledge of the solutions of the governing equations of the model (obtained often only with the assistance of computers), the validation with qualitative observations requires only knowledge of some qualitative properties of solutions. We shall see in Section 3 that Equation (1) displays manifestly an agreement with certain qualitative experimental observations.

\subsection{The Third Route to GENERIC: Thermodynamics}

The essential difference between the classical mechanics of particles and mesoscopic mechanics is that the former involves and keeps all of the details, while the latter ignores some of them. This ignorance enables one to recognize emerging overall features. How does the time evolution with and without all of the details differ from the mathematical point of view? It is thermodynamics, more precisely the classical equilibrium thermodynamics, where the answer to this question has started to emerge. The influence of 
the partial ignorance is seen in the time evolution of a new potential of non-mechanical nature that is called entropy. This potential tends to its maximum allowed by constraints (the so-called maximum entropy principle, abbreviated as the MaxEnt principle). Instead of the pure mechanical time evolution where permanence is the dominant feature, the mesoscopic (thermodynamic) time evolution emphasizes the asymmetry between beginning and end. As argued in [15], Aristotle's concept of the time evolution (introduced in his Physics) has common elements with the thermodynamic time evolution. Only much later, Galileo and Newton introduced the concept (and in this case, also the mathematical formulation) of the mechanic time evolution where the permanence and the time reversibility dominate.

In the mathematical structure of mesoscopic dynamics, we anticipate to find a certain reconciliation of the mechanic and the thermodynamic time evolution. The main focus should be put however on the thermodynamic time evolution, since the beginning-end asymmetry (seen also in mesoscopic experimental observations) remains to be its dominant and the most important feature. We would like to be able to see the mesoscopic dynamics as a natural extension of thermodynamics toward time evolution. We shall see in Section 4 that GENERIC (1) can indeed be seen in this way.

\subsection{What Physical Situations Are Described by GENERIC?}

Classical equilibrium thermodynamics, fluid mechanics, kinetic theory and classical mechanics of all microscopic particles composing macroscopic systems are an example of a sequence of four levels (also called scales) of the description of macroscopic systems. The first one is the most macroscopic among them (with the least number of details); the next one includes more details (it is a more microscopic level); and the fourth one is the most microscopic (it involves all possible details). The first level does not involve any time evolution; the remaining three do involve it. The dynamical (the static in the case of the first level) theories of macroscopic systems have arisen on all four levels from insight and experience collected in certain experimental observations (that are different for different levels). If we regard macroscopic systems on more than a single level, we say that our viewpoint is multilevel (or also multiscale).

We show in Section 3.2 below that solutions to (1) represent an approach of a level of description (we shall call it a starting level) to a more macroscopic level (that we shall call a target level). The states of the target level are states that maximize the entropy $S(x)$ subject to certain constraints (see also Example 3 in Section 4.3). If we choose the target level to be the level of equilibrium thermodynamics, then (1) describes the approach to thermodynamic equilibrium states. If the macroscopic systems under consideration are externally driven, then the level of equilibrium thermodynamics is not applicable, but in most cases, there is a mesoscopic level (i.e., a level that is more macroscopic than the level of particle mechanics) that is applicable. For example, let the system under consideration be the Rayleigh-Bénard system (a horizontal layer of fluid heated from below). The mesoscopic level on which such a system is found to be well described is the level of fluid mechanics. If we take this level as the target level and choose the starting level to be a more microscopic level (e.g., the level of kinetic theory), then (1), describing the approach of the kinetic theory level to the level of fluid mechanics, represents the application of GENERIC to the Rayleigh-Bénard problem (see more in Example 3 in Section 4.3). In this sense, the GENERIC Equation (1) applies to both closed systems (surrounded only by thermodynamic 
walls allowing one to change the volume and to pass the internal energy, the number of moles) and open externally-driven systems (e.g., systems discussed in biology). In the externally-driven systems, the GENERIC Equation (1) does not represent the time evolution of a driven system itself (e.g., the Boussinesq equations in the case of a Rayleigh-Bénard system), but it represents the complementary dynamics describing the approach seen on a more microscopic level (e.g., the level of kinetic theory in the case of a Rayleigh-Bénard system) to dynamics on the more macroscopic level on which the driven system is investigated (i.e., the level of fluid mechanics in the case of the Rayleigh-Bénard system). How does an investigation of the complementary dynamics contribute to the investigation of the driven system itself? It brings thermodynamics (in particular entropy) into the state space on which the driven system is investigated (see also Example 3 in Section 4.3).

\subsection{How Does GENERIC Relate to the Classical Nonequilibrium Thermodynamics?}

The classical nonequilibrium thermodynamics (that has been developed mainly on the level of fluid mechanics; see, e.g., [17]) is recovered as a particular realization of (1) on the level of fluid mechanics (with a supplementary extra structure, e.g., the Hamiltonian structure of the Euler part of the time evolution). Indeed, the classical nonequilibrium thermodynamics belongs to the pool of dynamical theories from which (1) has been extracted as a common structure (see the first paragraph in this section). GENERIC Equation (1) represents, as one particular example, the governing equations of the classical nonequilibrium thermodynamics. In particular, if (1) is linearized about the states reached as $t \rightarrow \infty$, then the Onsager reciprocity relations are recovered (see more about this point in [18] and [15]). The difference between the GENERIC viewpoint of nonequilibrium thermodynamics and the classical viewpoint is well demonstrated in the role that the Boltzmann kinetic theory plays in both viewpoints. In the classical nonequilibrium thermodynamics, the Boltzmann kinetic equation serves (see, e.g., [17]) as a microscopic basis from which the structure of the classical theory unfolds. From the point of view of GENERIC, the kinetic theory plays the same role, but it takes also an additional role. It is itself the nonequilibrium thermodynamics (another particular realization of (1)). The GENERIC time evolution Equation (1) represents nonequilibrium thermodynamics on any level of description.

Following Langevin, the dissipation is introduced in some approaches to mesoscopic dynamics by upgrading the status of state variables to random variables and by introducing into dynamics a noise. This formulation can be transformed into a formulation in which state variables $x$ become distribution functions $f(x)$ and the time evolution equation becomes a Fokker-Planck-type kinetic equation. It is then this formulation that becomes a particular realization of (1) (see more details in Section 3.1, Equation (9)).

\section{Mathematics of GENERIC}

We first complete the mathematical formulation of (1) and then investigate some qualitative properties of its solutions. 


\subsection{Mathematical Formulation of (1)}

The first term on the right-hand side of (1) represents the mechanic time evolution. The symbol $L$ is an operator transforming a covector (gradient of the energy $E(x)$ ) into a vector. We use hereafter the following notation. The state space is denoted by the symbol $M$ (i.e., $x \in M$ ); $B$ and $C$ are real-valued sufficiently-regular functions of $x$; and $<,>$ denotes the pairing in $M$. We define a bracket $\{A, B\}$ by $<A_{x}, L B_{x}>=\{A, B\}$. This bracket is called a Poisson bracket (or equivalently, $L$ is a Poisson bivector) if $\{A, B\}=-\{B, A\}$, and the Jacobi identity $\{\{A, B\} C\}+\{\{B, C\} A\}+$ $\{\{C, A\} B\}=0$ holds. Moreover, we say that the time evolution equation $\dot{x}=L E_{x}$ is Hamiltonian if $E: M \rightarrow \mathbb{R}$ has the physical interpretation of energy and $L$ is a Poisson bivector (in other words, if $<A_{x}, L B_{x}>=\{A, B\}$ is a Poisson bracket).

As an illustration, we take $x=(p, q)$ and $L=\left(\begin{array}{cc}0 & -1 \\ 1 & 0\end{array}\right)$. The time evolution equation $\dot{x}=L E_{x}$ (i.e., $\left(\begin{array}{c}\dot{p} \\ \dot{q}\end{array}\right)=\left(\begin{array}{c}-E_{q} \\ E_{p}\end{array}\right)$ ) is the equation governing the Hamiltonian time evolution of a classical particle with coordinate $q$ and momentum $p$.

The GENERIC Equation (1) without the second term on its right-hand side is required to be Hamiltonian, but in addition, it is also required to be compatible with the thermodynamic time evolution represented by the second term. The compatibility is defined by requiring $L S_{x}=0$, where $S(x)$ is the potential, called entropy, that makes its appearance in the second term. This compatibility condition can also be formulated as the degeneracy of the Poisson bracket: $\{A, S\}=0$ for all $A$. In the general theory of Hamiltonian systems, a function $S$ satisfying the above relation is called a Casimir. By using this terminology, we thus require that the GENERIC equation $\dot{x}=L E_{x}$ is Hamiltonian with the entropy $S$ (appearing in the second term $\left[\chi \frac{\partial \Xi\left(x, x^{*}\right)}{\partial x^{*}}\right]_{x^{*}=S_{x}}$ ) as its Casimir.

A simple illustration of a Hamiltonian time evolution that is in addition compatible with the thermodynamic time evolution is provided by Equation (2). In order to prove it, it suffices to make a transformation $(p, q, e) \rightarrow(p, q, S)$, where $p=p ; q=q ; S=S(p, q, e)$. This transformation is one-to-one as a consequence of the assumption $S_{e}>0$ (since we interpret physically $S_{e}$ as inverse of the absolute temperature). It is easy to see that the matrix $L$ transforms into $\left(\begin{array}{ccc}0 & -1 & 0 \\ 1 & 0 & 0 \\ 0 & 0 & 0\end{array}\right)$, which is manifestly a Poisson bivector, and $S$ is its Casimir.

The Poisson brackets corresponding to the Boltzmann kinetic equation and to the governing equations of fluid mechanics will be recalled later in this section.

Now, we turn to the second term on the right-hand side of (1) representing the compatible-with-mechanics thermodynamic time evolution. We recall that the symbol $M$ stands for the state space $(x \in M)$. By $M^{*}$, we denote its dual $\left(x^{*} \in M^{*}\right)$. The quantity $\chi: M \times M^{*} \rightarrow \mathbb{R}^{+}$is required to be positive; otherwise, we leave it unspecified. The function $\Xi: M \times M^{*} \rightarrow \mathbb{R}$, called a dissipation potential, is required to satisfy the following properties: 


$$
\begin{aligned}
& \Xi(x, 0)=0, \forall x \in M \\
& \Xi \text { reaches its minimum at } x^{*}=0 \\
& \Xi \text { is a convex function in a neighbourhood of } x^{*}=0
\end{aligned}
$$

We shall first consider a special case when $\Xi$ is a quadratic function of $x^{*}$, i.e.,

$$
\Xi\left(x, x^{*}\right)=\frac{1}{2}<x^{*}, \Lambda(x) x^{*}>
$$

where $\Lambda(x)$ is a positive definite operator transforming a covector $x^{*}$ into a vector. For this dissipation potential, the second term on the right-hand side of (1) takes the form $\chi \Lambda(x) S_{x}$. The compatibility with the mechanic time evolution is defined as a degeneracy of $\Lambda$. We require that $\Lambda(x) E_{x}=0$ for all $x \in M$. The definition of the compatibility of the thermodynamic time evolution with the mechanic one is thus the same as the definition of the compatibility of the mechanic time evolution with the thermodynamic one. Only the roles of energy and entropy are exchanged. In the former, $S(x)$ is the generating potential, and the compatibility requirement is a degeneracy of the operator transforming the gradient of $S(x)$ into a vector (i.e., the requirement $\Lambda E_{x}=0$ ). In the latter, it is $E(x)$ that plays the role of the generating potential, and the compatibility requirement is a degeneracy of the operator $L$, transforming the gradient of $E(x)$ into a vector (i.e., the requirement $L S_{x}=0$ ).

For a general dissipation potential $\Xi$, we define the compatibility with mechanics by $<E_{x},\left[\Xi_{x^{*}}\right]_{x^{*}=S_{x}}>=0$ and $<x^{*},\left[\Xi_{x^{*}}\right]_{x^{*}=E_{x}}>=0, \forall x^{*}$.

For the sake of generality, we may also require additional degeneracy in both mechanic and thermodynamic time evolution. Let:

$$
W: M \rightarrow \mathbb{R}
$$

be a sufficiently regular function (or functions). The additional degeneracy is defined by $L W_{x}=0$ and $\Lambda W_{x}=0$ or, in the case of the general dissipation potential, $L W_{x}=0,<W_{x},\left[\Xi_{x^{*}}\right]_{x^{*}=S_{x}}>=0$ and $<x^{*},\left[\Xi_{x^{*}}\right]_{x^{*}=W_{x}}>=0, \forall x^{*}$. Examples of $W$ are discussed in Section 4.1.

We end this section by recalling the Poisson brackets and dissipation potentials with which (1) becomes the Boltzmann kinetic equation and the governing equations of fluid mechanics. We begin with Poisson brackets.

In kinetic theory, the state variable $x$ is one particle distribution function $f(\boldsymbol{r}, \boldsymbol{v})$, where $\boldsymbol{r} \in \mathbb{R}^{3}$ is the position vector and $\boldsymbol{v} \in \mathbb{R}^{3}$ is the momentum. The Poisson bracket $\{A, B\}^{(f)}$ expressing kinematics of $f(\boldsymbol{r}, \boldsymbol{v})$ is given by:

$$
\{A, B\}^{(f)}=\int d \boldsymbol{r} \int d \boldsymbol{v} f\left\{A_{f}, B_{f}\right\}^{(\mathrm{cm})}
$$

where $A: M \rightarrow \mathbb{R}, A_{f(\boldsymbol{r}, \boldsymbol{v})}=\frac{\delta A}{\delta f(\boldsymbol{r}, \boldsymbol{v})}$ is the appropriate functional derivative (similarly, $B_{f}$ is an appropriate functional derivative of $B: M \rightarrow \mathbb{R}$ ) and:

$$
\{A, B\}^{(\mathrm{cm})}=\left(A_{r}, A_{v}\right) L^{(\mathrm{cm})}\left(\begin{array}{c}
B_{r} \\
B_{v}
\end{array}\right)
$$


is the canonical Poisson bracket of particle mechanics (the superscript $(\mathrm{cm})$ stands for classical mechanics); $L^{(\mathrm{cm})}=\left(\begin{array}{cc}0 & 1 \\ -1 & 0\end{array}\right)$ and $A, B$ in (7) are functions $\mathbb{R}^{6} \rightarrow \mathbb{R}$ and $A_{r}=\frac{\partial A}{\partial \boldsymbol{r}}$, similarly $A_{v}, B_{r}, B_{v}$. The bracket (6) has arisen in the works of $[2,3,20]$ as a bracket that is canonically associated with a Lie group of transformations representing the mechanical motion (in this case, the transformations are the canonical transformations in the six-dimensional space with coordinates $(\boldsymbol{r}, \boldsymbol{v})$ ). It can easily be verified that the bracket (6) is degenerate; it has an infinite number of Casimirs (that are functions $\int d \boldsymbol{r} \int d \boldsymbol{v} c(f(\boldsymbol{r}, \boldsymbol{v}))$, where $\left.c: \mathbb{R} \rightarrow \mathbb{R}\right)$.

In fluid mechanics, the state variables are hydrodynamic fields: $x=(\rho(\boldsymbol{r}), \boldsymbol{u}(\boldsymbol{r}), s(\boldsymbol{r}))$ denoting the fields of mass momentum end entropy per unit volume. Their kinematics is mathematically expressed in the Poisson bracket:

$$
\begin{array}{r}
\{A, B\}=\int d \boldsymbol{r}\left(\begin{array}{lll}
A_{\rho}, & A_{s}, & A \boldsymbol{u}
\end{array}\right) L\left(\begin{array}{c}
B_{\rho} \\
B_{s} \\
B \boldsymbol{u}
\end{array}\right) \\
=\int d \boldsymbol{r}\left[\rho\left(\partial_{i}\left(A_{\rho}\right) B_{u_{i}}-\partial_{i}\left(B_{\rho}\right) A_{u_{i}}\right)\right. \\
+s\left(\partial_{i}\left(A_{s}\right) B_{u_{i}}-\partial_{i}\left(B_{s}\right) A_{u_{i}}\right) \\
\left.+u_{j}\left(\partial_{i}\left(A_{u_{j}}\right) B_{u_{i}}-\partial_{i}\left(B_{u_{j}}\right) A_{u_{i}}\right)\right]
\end{array}
$$

where we use again the same abbreviated notation (a symbol in the position of the lower index denotes the derivative with respect to the quantity represented by the symbol), $\partial_{i}=\frac{\partial}{\partial r_{i}} ; i=1,2,3$; and we use the summation convention. This bracket can again be derived from its canonical association (see [2,3]) with the Lie group representing the motion of continuum (in this case, it is the group of transformations $\mathbb{R}^{3} \rightarrow \mathbb{R}^{3}$ ). It can however also be derived in, at least, two other ways, namely as a symmetry reduction (see [20]) of the canonical Poisson bracket representing the Lagrangian view of the motion of continuum (as a motion of fluid particles, the symmetry group is the group of relabelling the fluid particles) and from the bracket (6) (see the details in [21]) by simply restricting the functions $A(f)$ and $B(f)$ appearing in it to those that depend on $f(\boldsymbol{r}, \boldsymbol{v})$ only through their dependence on the moments $\rho(\boldsymbol{r})=\int d \boldsymbol{v} f(\boldsymbol{r}, \boldsymbol{v}) ; \boldsymbol{u}(\boldsymbol{r})=\int d \boldsymbol{v} \boldsymbol{v} f(\boldsymbol{r}, \boldsymbol{v}) ; s(\boldsymbol{r})=\int d \boldsymbol{v} c(f(\boldsymbol{r}, \boldsymbol{v})) f(\boldsymbol{r}, \boldsymbol{v})$ (where $c(f)$ is the Casimir density introduced in the previous paragraph) expressing the hydrodynamic fields.

Now, we turn to dissipation potentials. In the Boltzmann kinetic equation, the source of dissipation is the ignorance of the details of the pieces of trajectories of the particles (the particles composing the macroscopic system under consideration) in the events of collision. The collision is seen as a chemical reaction in which two species of particles labelled by the momenta $\boldsymbol{v}$ and $\boldsymbol{v}_{1}$ undergo a binary collision (a binary chemical reaction) whose outcomes are two species labelled by the momenta $\boldsymbol{v}^{\prime}$ and $\boldsymbol{v}_{1}^{\prime}$. The relation between $\left(\boldsymbol{v}, \boldsymbol{v}_{1}\right)$ and $\left(\boldsymbol{v}^{\prime}, \boldsymbol{v}_{1}^{\prime}\right)$ is only constrained by the conservation of the total momenta and the total kinetic energy. Following chemical kinetics [22], we thus have $\Xi=\int d \boldsymbol{v} \int d \boldsymbol{v}_{1} \int d \boldsymbol{v}^{\prime} \int d \boldsymbol{v}_{1}^{\prime} W\left(f ; \boldsymbol{v}, \boldsymbol{v}_{1}, \boldsymbol{v}^{\prime}, \boldsymbol{v}_{1}^{\prime}\right)\left(e^{\frac{1}{2} X}+e^{-\frac{1}{2} X}-2\right)$, where $W$ is different from zero and positive only if $\boldsymbol{v}+\boldsymbol{v}_{1}=\boldsymbol{v}^{\prime}+\boldsymbol{v}_{1}^{\prime}$ and $v^{2}+v_{1}^{2}=\left(v^{\prime}\right)^{2}+\left(v^{\prime}\right)_{1}^{2}, W$ is symmetric with respect to the exchanges $\boldsymbol{v} \leftrightarrows \boldsymbol{v}_{1}$ and $\left(\boldsymbol{v}, \boldsymbol{v}_{1}\right) \leftrightarrows\left(\boldsymbol{v}^{\prime}, \boldsymbol{v}_{1}^{\prime}\right)$, and $\boldsymbol{X}\left(\boldsymbol{r}, \boldsymbol{v}, \boldsymbol{v}_{1}, \boldsymbol{v}^{\prime}, \boldsymbol{v}_{1}^{\prime}\right)=-f^{*}(\boldsymbol{r}, \boldsymbol{v})-f^{*}\left(\boldsymbol{r}, \boldsymbol{v}_{1}\right)+f^{*}\left(\boldsymbol{r}, \boldsymbol{v}^{\prime}\right)+f^{*}\left(\boldsymbol{r}, \boldsymbol{v}_{1}^{\prime}\right)$ is the chemical affinity (for details, see [21]). The required degeneracy of this dissipation potential is easily verified. 
In the setting of fluid mechanics, in order to construct a dissipation potential with the required degeneracy, we make first a one-to-one transformation in the state variables. We pass from the hydrodynamic fields $(\rho(\boldsymbol{r}), \boldsymbol{u}(\boldsymbol{r}), s(\boldsymbol{r}))$ to the hydrodynamic fields $(\rho(\boldsymbol{r}), \boldsymbol{u}(\boldsymbol{r}), e(\boldsymbol{r}))$, where $e(\boldsymbol{r})$ is the energy field (the transformation is indeed one-to-one, since $\frac{\partial e}{\partial s}$, having the physical interpretation of the local temperature, is positive). The dissipation potential leading to the Navier-Stokes-Fourier part of the fluid mechanics equations is the potential that is quadratic in two thermodynamic forces: the Navier-Stokes force $\boldsymbol{X}^{(N S)}=\overline{\nabla\left(\frac{\boldsymbol{u}^{*}}{e^{*}}\right)}$ and the Fourier force $\boldsymbol{X}^{(F)}=\nabla\left(\frac{1}{e^{*}}\right)$. By $\overline{\boldsymbol{a}}$, we denote a symmetric part of the tensor $\boldsymbol{a}$. The required degeneracy is again easily verified, the multiplicative factor $\chi$ is in this case $e^{*}$ (see the details in [21]).

In the previous section, we have mentioned the Langevin lift of (1) that itself is a particular realization of (1). We recall that, from the physical point of view, the Langevin lift is an extension to a more detailed formulation in which fluctuations are taken into consideration. In the context of GENERIC, we make the Langevin lift as follows. We make the following modifications in the quantities entering (1) (the superscript FP stands for Fokker-Planck): $x \mapsto x^{F P}=f(x)$ denoting the distribution function of $x, E(x) \mapsto E^{F P}(f)=\int d x f(x) E(x) ;\{A(x), B(x)\} \mapsto\left\{A^{F P}(f), B^{F P}(f)\right\}^{F P}=$ $\int f(x)\left\{A_{f(x)}^{F P}(f), B_{f(x)}^{F P}(f)\right\} ; S(x) \mapsto S^{F P}(f)=\int d x f(x)\left(S(x)-k_{B} \ln f(x)\right)$, where $k_{B}$ is the Boltzmann constant, and if we restrict ourselves only to quadratic dissipation potential (4), $\Xi\left(x, S_{x}\right) \mapsto$ $\left.\Xi^{F P}\left(f, S_{f}^{F P}\right)=\int d x f(x)<\frac{\partial}{\partial x}\left(S_{f(x)}^{F P}\right)\right) \Lambda \frac{\partial}{\partial x}\left(S_{f(x)}^{F P}\right)>$. With these changes, the GENERIC Equation (1) becomes:

$$
\frac{\partial f(x)}{\partial t}=-\frac{\partial}{\partial x}\left(f(x) L \frac{\partial E(x)}{\partial x}\right)-\frac{\partial}{\partial x}\left(f(x) \Lambda \frac{\partial S(x)}{\partial x}\right)+\frac{\partial}{\partial x}\left(f(x) \Lambda \frac{\partial f(x)}{\partial x}\right)
$$

We note that without the last term on the right-hand side, Equation (9) is simply the Liouville equation corresponding to (1). The last term on the right-hand side is a new diffusion term that arises due to fluctuations. We see that its origin is the new term $-k_{B} \int d x f(x) \ln f(x)$ that we have added to the entropy. The fact that the same matrix $\Lambda$ appears in the second and the third term on the right-hand side of (9) is a statement of the fluctuation-dissipation theorem. In this derivation, we see very clearly that the theorem is a direct consequence of the requirement of the compatibility of the Fokker-Planck-type Equation (9) with thermodynamics in the sense that the Fokker-Plank kinetic equation is a particular realization of the GENERIC Equation (1), and consequently, as we shall see below, its solutions obey the relations (10)-(12).

\subsection{Properties of Solutions of (1)}

It follows directly from the above mathematical formulation of (1) that:

$$
\begin{aligned}
& \frac{d E}{d t}=0 \\
& \frac{d S}{d t} \geq 0 \\
& \frac{d W}{d t}=0
\end{aligned}
$$

The first equality is a consequence of the fact the the energy $E(x)$ generates the mechanical (Hamiltonian) part of (1) (i.e., from $\frac{d E}{d t}=\{E, E\}=0$ ) and from the required degeneracy of its 
thermodynamic part. The second inequality follows from the required degeneracy of the mechanical part of the time evolution and from the properties (3) of the dissipation potential. The third equality is a consequence of the required degeneracy of both the mechanic and the thermodynamic parts of the time evolution generated by (1). From the physical point of view, the relations (10)-(12) mean that in the course of the time evolution generated by (1), the total energy and other quantities represented mathematically by $W$ are conserved and the total entropy $S(x)$ does not decrease.

Another property of solutions of (1) that is a direct consequence of (10)-(12) and that is seen in experimental observations is the approach to equilibrium at which the classical equilibrium thermodynamics applies. We note that the relation (10)-(12) implies that $\mathcal{L}(x)=-S(x)+k^{(E)} E(x)+$ $k^{(W)} W(x)$ plays the role of the Lyapunov function corresponding to the approach to states at which $\mathcal{L}$ reaches its minimum provided $\mathcal{L}$ is convex; $k^{(E)}$ and $k^{(W)}$ are constants. We shall investigate this connection with thermodynamics in detail in Section 4.

We mention still two other properties of solutions of (1). We begin with the existence and regularity of solutions. The physical processes described by the governing equations are seen in experimental observations to exist, they should therefore exist also as solutions to the equations. This basic requirement of agreement between results of observations and theoretical predictions is however rarely proven. The mathematical proof of the existence of solutions requires a detailed specification of Equation (1) that goes beyond the specifications made above. For example, the topology has to be explicitly specified. The question then arises: what is the physical interpretation of the topology? Since the topology is a mathematical formulation of "closeness", the answer should depend on the way the experimental observations are actually made (which states appearing to be close, or closer, from the point of view of observing them experimentally). We recall that a proof of the existence and regularity of solutions to the particular realization of (1) that represents the Boltzmann kinetic equation has appeared only recently (in 1991) in [23]. Physical interpretation of the mathematical specifications needed in the proof remains, to the best of our knowledge, an open problem. We shall make another comment about the relation between physics and proofs of existence of solutions in Section 3.3 below.

The second property is about the interplay between the mechanic vector field (the first term on the right-hand side of (1)) and the thermodynamic vector field (the second term on the right-hand side of (1)) in the time evolution that they both generate. The result that we want to recall is, roughly speaking, the following. The mechanic vector field (even if by itself completely non-dissipative) makes, in the course of the time evolution, the thermodynamic vector field more dissipative. First, we explain what we mean by a less or a more dissipative vector field. We say that a vector field $\mathbb{V}_{1}$ is more dissipative than $\mathbb{V}_{2}$ if the manifold $\mathcal{M}_{1}$ to which solutions to $\frac{d x}{d t}=\mathbb{V}_{1}(x)$ approach as $t \rightarrow \infty$ (i.e., the manifold obtained as a solution to $\left.\mathbb{V}_{1}(x)=0\right)$ is a submanifold of the manifold $\mathcal{M}_{2}$ to which solutions to $\frac{d x}{d t}=\mathbb{V}_{2}(x)$ approach as $t \rightarrow \infty$ (i.e., the manifold obtained as a solution to $\mathbb{V}_{2}(x)=0$ ).

We continue to discuss the interplay between mechanic and thermodynamic vector fields only in the setting corresponding to the Boltzmann kinetic equation, since in this particular setting, the problem has been studied in detail [24,25]. In the absence of the mechanic vector field (i.e., $-\frac{\partial}{\partial \boldsymbol{r}}\left(f \frac{\boldsymbol{v}}{m}\right)$ ), solutions to the Boltzmann equation approach, as $t \rightarrow \infty$, states of local equilibrium (that is, a manifold in the state space $M ; f(\boldsymbol{r}, \boldsymbol{v}) \in M$ constructed as an image of the embedding $(\rho(\boldsymbol{r}), \boldsymbol{u}(\boldsymbol{r}), e(\boldsymbol{r})) \hookrightarrow M$ that is obtained by solving $\boldsymbol{X}\left(\boldsymbol{r}, \boldsymbol{v}, \boldsymbol{v}_{1}, \boldsymbol{v}^{\prime}, \boldsymbol{v}_{1}^{\prime}\right)=-f^{*}(\boldsymbol{r}, \boldsymbol{v})-f^{*}\left(\boldsymbol{r}, \boldsymbol{v}_{1}\right)+f^{*}\left(\boldsymbol{r}, \boldsymbol{v}^{\prime}\right)+f^{*}\left(\boldsymbol{r}, \boldsymbol{v}_{1}^{\prime}\right)=$ 
0 ). On the other hand, in the absence of the thermodynamic vector field (i.e., in the absence of the Boltzmann collision term), the time evolution proceeds as $f(\boldsymbol{r}, \boldsymbol{v}) \mapsto f\left(\boldsymbol{r}-\frac{\boldsymbol{v}}{m} t, \boldsymbol{v}\right)$, and the entropy remains constant. If both vector fields are present, then solutions approach the total equilibrium (that is, a small submanifold of the local equilibrium corresponding to $(\rho(\boldsymbol{r}), \boldsymbol{u}(\boldsymbol{r}), e(\boldsymbol{r}))$, which are independent of $\boldsymbol{r}([24,25])$. This means that by adding to the Boltzmann collision vector field another vector field, which, by itself, is completely non-dissipative, we obtain a vector field that is more dissipative than the original Boltzmann collision vector field. This is a very important result that tells us, for example, that separate investigations of the mechanic and thermodynamic vector fields represent only a small part of investigations of solutions to (1).

This result about the interplay between mechanic and thermodynamic vector fields motivates also the following conjecture about the emergence of dissipativity and time irreversibility. Let us begin to investigate the time evolution of a macroscopic system by keeping all of the details (i.e., we follow completely trajectories of all particles composing it). Such a time evolution is time reversible and non-dissipative. Now, we decide to ignore a small detail (it could be, for example, that we decide to consider the initially distinguishable particles as indistinguishable). In other words, we decide to introduce a small grain of dissipativity and time irreversibility. We conjecture that due to the interplay of this newly-introduced small thermodynamic vector field with the rest of the vector field, this grain then grows by itself. No additional interventions are needed to increase the dissipativity to the level that is needed to bring externally unforced macroscopic systems to equilibrium states at which the classical equilibrium thermodynamics applies.

\subsection{Other Versions of Equation (1)}

As we have already noted in Section 2, we cannot claim that the mathematical structure collected in (1) is the only common structure seen in mesoscopic dynamical theories. Indeed, several versions of (1) have been suggested. We present some of them below.

First, we turn to the mechanic vector field (i.e., to the first term on the right-hand side of (1)). As we have already mentioned, its mathematical structure has unfolded from the Clebsch observation [1] about the Euler vector field arising in fluid mechanics (that represents a particular realization of the mechanic vector field). The Hamiltonian structure recognized in it by Clebsch has been then recognized also in mechanic vector fields arising in other mesoscopic dynamical theories, and it has been subsequently elevated to the status of an abstract structure representing the mechanics in mesoscopic dynamics. There is, however, also another path starting at the Euler vector field and ending with another abstract mathematical structure. We shall now briefly follow it.

The state variables in the Euler fluid mechanics are fields (i.e., functions of the position vector $\boldsymbol{r}$ ), and the time evolution equations are local conservation laws (i.e., the vector fields are gradients of a flux that is a pointwise function of the fields serving as state variables). We now elevate this feature of the Euler vector field to an abstract setting. This is then the setting discussed by Godunov [26-28]. We make two observations. 
First, we note that the Euler dynamics possesses both the Hamiltonian and the Godunov structure. How do these two structures relate in the abstract setting? To the best of our knowledge, the answer to this question is not known.

Second, we look at the degeneracy. In the setting of the Godunov structure, the degeneracy (which, in the setting of the Hamiltonian structure, corresponds to the requirement that entropy is Casimir of the Poisson bracket) is a requirement that the equation governing the time evolution of the entropy field (that is, a pointwise function of the fields included into state variables) is also a local conservation law. It turns out that this requirement motivated by physics has important consequences for the existence and regularity of solutions to the Godunov dynamical equations (see [26,28,29]). Godunov dynamics provides thus a rare example of a direct relation between physical clarity and clarity of the mathematical formulation.

Now, we turn to the thermodynamic vector field (i.e., to the second term on the right-hand side of (1)). Here, we find even larger variety. The original model from which all variations unfolded is the Ginzburg-Landau-Cahn-Hilliard vector field (see (4)). A necessity to generalize the Ginzburg-Landau-Cahn-Hilliard vector field has arisen probably most clearly in chemical kinetics. In order to put the thermodynamic vector field that arises in chemical kinetics into the form (4), we need either to pass to convex dissipation potentials (as we do in Section 3; see also [22] and Section 4.2 below) or to allow the operator $\Lambda$ appearing in (4) to depend also on $x^{*}$ (see [30]). We note already at this point (see more in Section 4.2 below) that the latter generalization destroys the geometrical content of $\Lambda$ (as an operator transforming a covector into a vector). The geometrical content can be preserved in a particular case and in a particular reformulation discussed in [31]. A very systematic and physically well-motivated approach to thermodynamic vector fields has recently been developed in [32]. At this point, we emphasize again the observation that we made at the end of Section 3.2, namely that a separate investigation of thermodynamic and mechanic vector fields provides very incomplete information about mesoscopic dynamics.

\section{Geometry of GENERIC}

Our objective in this section is to present the GENERIC Equation (1) as an integral part of general thermodynamics. As for the language that we intend to employ, we choose the language of geometry, since, as we known from many other fields of physics, it is the most appropriate language to express physics. In particular, we look for guidance in the geometrical formulation of mechanics (see, e.g., [33]).

What is missing in (1), and what do we anticipate to achieve? If the second term on the right-hand side of (1) were absent, then (1) expresses only mechanics, and the geometry that provides an excellent environment to formulate it is well known. It is symplectic geometry. However, in the presence of the second term, the symplectic geometry ceases to play such a role. This is because the symplectic structure, playing in mechanics the role of a structure (physically interpreted as a structure expressing kinematics) transforming forces (covectors obtained as gradients of energy) into vectors, is no longer preserved during the time evolution. Moreover, there is another structure, namely the Riemannian structure (or some of its generalizations), that transforms other covectors (that can be seen as entropic forces, also called thermodynamic forces, obtained as gradients of another potential that is called entropy) into 
vectors. What is the geometrical environment in which both terms on the right-hand side of (1) are getting a clear and a unified geometrical interpretation? We expect that such an environment will also be, from the physical point of view, an environment that is appropriate to formulate thermodynamics. We therefore begin with a geometrical formulation of classical thermodynamics.

\subsection{Geometry of Thermodynamics}

We recall first the physics of the classical thermodynamics. All of the information coming from microscopic dynamics, except the conservations of mass and energy and the ergodicity-type behaviour of microscopic trajectories (vaguely formulated as the equality of time and certain microscopic state space averages), is ignored in this theory. The state variables are only the quantities that characterize overall features, namely the volume $V$, the mass (or alternatively, the number of moles $N_{m o l}$ ) and the energy $E$. The energy is then split into two parts: one, denoted $E_{\text {macro }}$, is associated with the overall behaviour, and the rest, called an internal energy $E_{i n t}$, is the energy associated with the microscopic details that do not enter explicitly the formulation. The total energy $E=E_{\text {macro }}+E_{\text {int }}$. The energy $E_{\text {macro }}$ passes through walls surrounding the macroscopic system under investigation as mechanical work and the internal energy as heat. The separation of the total energy into two parts and its conservation is the subject of the so-called "first law of thermodynamics".

However, this formulation cannot be complete, since the details of microscopic dynamics that are ignored still influence the macroscopic behaviour. There has to be therefore a way to express this type of influence without the details that we do not want to have explicitly included in the formulation. The way this is done is the most important and the most original (original with respect to mechanics) contribution of thermodynamics. It is called the "second law of thermodynamics". The influence of ignored microscopic details is expressed in a new potential that is called entropy and is usually denoted by the symbol $S$. This new potential is a real valued function of $(E, N, V)$. It is in the function $S=S(E, N, V)$, where the individual nature of macroscopic systems is expressed in the classical equilibrium thermodynamics. The ignored microscopic details are influencing the behaviour of macroscopic systems in such a way that the entropy reaches always its maximum allowed by macroscopic constraints. The tendency of $S$ to reach the maximum is called the "maximum entropy principle" (MaxEnt; see [34,35]).

How shall we formulate mathematically (and geometrically) this new mechanics with ignored details? Among many suggestions that have been advanced, we turn to the one proposed by Robert Hermann [36] (see also [37]). We make this choice, since his formulation naturally extends (as we shall see later in this section) to mesoscopic and microscopic levels with the varying amount of ignored details and also to the time evolution governed by (1) (as we shall see in Section 4.2).

One sequence of arguments leading to Hermann's formulation is the following. Being inspired by the arguments developed mainly in elementary physics, we ask two questions. The first one is of what is the group of transformations that plays the most important role in thermodynamics. We answer it by saying that it is the group of Legendre transformations. This is because the essence of thermodynamics is the MaxEnt principle, and maximization with constraints is, from the mathematical point of view, a Legendre transformation. Besides, we recall that any presentation of thermodynamics, ranging from 
those directed mainly to engineering applications (e.g., [38]) to those directed mainly to physicists ([39]), recognizes the importance of Legendre transformations in both the explanation of the physics involved and in applications.

Having answered the first question, we ask the second question: What is the most natural geometrical environment for the Legendre transformations? The answer is well known: it is the environment provided by contact geometry (see, e.g., [33]).

Equilibrium thermodynamics is presented in four steps. In every step, we provide two illustration, one corresponding to the classical equilibrium thermodynamics and the second to kinetic theory.

\section{Step 1: Starting and target levels; state spaces $M$ and $N$}

Equilibrium thermodynamics involves always two levels of description, one on which we see the macroscopic system under investigation initially (we shall call it a starting level) and the other (called a target level) that is reached by maximizing the entropy. The state space on the starting level will be denoted by the symbol $M$ and its elements by $x$ (i.e., $x \in M$ ). On the target level, the state space is $N$ and its elements $y$ (i.e., $y \in N$ ).

Illustration: Classical equilibrium thermodynamics

In this example, both the starting and target levels are the same, i.e., $M \equiv N$. The state variables are $x=(e, n)$, denoting the energy and the number of moles per unit volume (for the sake of brevity, we shall put hereafter the volume equal to one).

\section{Illustration: Kinetic theory}

The state variable $x=f(\boldsymbol{r}, \boldsymbol{v})$ is the one particle distribution function; $\boldsymbol{r}$ is the position coordinate and $\boldsymbol{v}$ momentum of one particle. The target space we choose in this illustration to be the state space of the classical equilibrium thermodynamics, i.e., $y=(e, n)$.

\section{Step 2: Fundamental thermodynamic relation}

We begin to equip the spaces $M$ and $N$ with a structure. First, we introduce the fundamental thermodynamic relation:

$$
\begin{gathered}
S: M \rightarrow \mathbb{R} ; x \mapsto S=S(x) \\
\mathcal{P}: M \rightarrow N ; x \mapsto y=\mathcal{P}(x)
\end{gathered}
$$

The function $S$ is assumed to be sufficiently regular and concave; the mapping $\mathcal{P}$ is assumed to be sufficiently regular and onto it.

\section{Illustration: Classical equilibrium thermodynamics}

It is in the function $S$ where the individual nature of macroscopic systems is expressed in the classical equilibrium thermodynamics. The mapping $\mathcal{P}$ is the identity mapping, i.e., $e \mapsto e$ and $n \mapsto n$.

\section{Illustration: Kinetic theory}

Following Boltzmann, we choose $S(f)=-k_{B} \frac{1}{h^{3}} \int d \boldsymbol{r} \int d \boldsymbol{v} f(\boldsymbol{r}, \boldsymbol{v})(\ln f(\boldsymbol{r}, \boldsymbol{v})-1)$ and $\mathcal{P}(f)=$ $\left(\int d \boldsymbol{r} \int d \boldsymbol{v} \frac{\boldsymbol{v}^{2}}{2 m} f(\boldsymbol{r}, \boldsymbol{v}), \int d \boldsymbol{r} \int d \boldsymbol{v} f(\boldsymbol{r}, \boldsymbol{v})\right)$, where $k_{B}$ is the Boltzmann constant, $m$ the mass of one particle, and $h$ is Planck's constant. 


\section{Step 3: State space $\mathbb{M}^{(\mathbb{N})}$ equipped with the contact structure}

In order to provide a setting that is suitable for the MaxEnt principle, we construct from the spaces $M, N$ and from their conjugates $M^{*}, N^{*}$ a new space $\mathbb{M}^{(\mathbb{N})}=M \times M^{*} \times N^{*} \times N \times \mathbb{R}$ with coordinates $\left(x, x^{*}, y^{*}, y, \phi\right)$. The elements of $M^{*}$ and $N^{*}$ are denoted $x^{*}$ and $y^{*}$, the inner product in both space $M$ and $N$ is denoted by $<,>$. The space $\mathbb{M}^{(\mathbb{N})}$ is naturally equipped with the completely non-integrable one-form:

$$
\vartheta=d \phi-x^{*} d x-y d y^{*}
$$

which brings to $\mathbb{M}^{(\mathbb{N})}$ the contact structure. We recall that if $M$ and $N$ are finite dimensional spaces with dimensions $m$ and $n$, respectively, then a one-form $\vartheta$ in $\mathbb{M}^{(\mathbb{N})}$ is completely non-integrable if the maximum dimension of a manifold on which $\vartheta=0$ is $m+n$.

Next, we introduce a new potential combining the entropy $S$ and the mapping $\mathcal{P}$ that both have arisen in the fundamental thermodynamic relation (13) and (14):

$$
\Phi\left(x, y^{*}\right)=-S(x)+<y^{*}, \mathcal{P}(x)>
$$

\section{Step 4: Gibbs-Legendre manifold}

We are now in a position to represent the fundamental thermodynamic relation (13) and (14) geometrically as:

$$
\begin{aligned}
\mathcal{M}^{(\mathcal{N})}= & \left\{\left(x, x^{*}, y^{*}, y, \phi\right) \in \mathbb{M}^{(\mathbb{N})} \mid x^{*}=\Phi_{x}(x), y=\Phi_{y^{*}}, \phi=\Phi(x)\right\} \\
& \left(x, y^{*}\right) \hookrightarrow\left(x, \Phi_{x}\left(x, y^{*}\right), y^{*}, \Phi_{y^{*}}\left(x, y^{*}\right), \Phi\left(x, y^{*}\right)\right)
\end{aligned}
$$

On this manifold, the one-form $\vartheta$ equals zero. We call the manifold $\mathcal{M}^{(\mathcal{N})} \subset \mathbb{M}^{(\mathbb{N})}$ a Gibbs-Legendre manifold, since it combines the Gibbs manifold and the Legendre manifold. The Gibbs manifold is in the classical equilibrium thermodynamics a well-established name for a two-dimensional manifold embedded in $\mathbb{R}^{3}$ by the mapping $(e, n) \hookrightarrow(e, n, S(e, n))$, and the Legendre manifold is in contact geometry a well-established name for the manifold on which the contact one-form vanishes.

We note that in various projections of the GLmanifold, we see geometrically all of the elements of the maximization of entropy subjected to constraints. Namely:

(i) The restriction of the manifold $\mathcal{M}^{(\mathcal{N})}$ to $y^{*}=0$, i.e., the manifold $\left.\mathcal{M}^{(\mathcal{N})}\right|_{y^{*}=0}$ displays the fundamental thermodynamic relation (13), (14). Indeed, this manifold is the image of the mapping $x \hookrightarrow\left(x,-S_{x}(x), 0, \mathcal{P}(x),-S(x)\right)$.

(ii) The restriction of the manifold $\mathcal{M}^{(\mathcal{N})}$ to $x^{*}=0$, i.e., the manifold $\left.\mathcal{M}^{(\mathcal{N})}\right|_{x^{*}=0}$ displays the states that represent in $M$ the states in $N$ (we shall call them equilibrium states and denote them by the symbol $x_{e q}\left(y^{*}\right)$ ) at which the entropy $S$ reaches its maximum allowed by constraints $\mathcal{P}(x)$ and also the fundamental thermodynamic relation in $N$ implied by the fundamental thermodynamic relation (13) in $M$. Indeed, the manifold $\left.\mathcal{M}^{(\mathcal{N})}\right|_{x^{*}=0}$ is the image of the mapping $\left(x, y^{*}\right) \hookrightarrow$ $\left(x_{e q}\left(y^{*}\right), 0, y^{*}, \mathcal{P}\left(x_{e q}\left(y^{*}\right)\right), S^{*}\left(y^{*}\right)\right)$. The relation $S^{*}=S^{*}\left(y^{*}\right)$ is the Legendre transformation of the fundamental thermodynamic relation $S=S(y)$ in $N$. In other words, we arrive at $S=S(y)$ from $S^{*}=S^{*}\left(y^{*}\right)$ in three steps: first, we construct a potential $\Psi\left(y^{*}, y\right)=-S^{*}\left(y^{*}\right)+<y^{*}, y>$; second, we obtain $y^{*}(y)$ as solutions to the equation $\Psi_{y^{*}}=0$; and third, we evaluate $\Psi$ at $y^{*}(y)$, i.e., $S(y)=\Psi\left(y^{*}(y), y\right)$. 


\section{Illustration: Classical equilibrium thermodynamics}

In the notation of the classical equilibrium thermodynamics, $\left.\left(e^{*}, n^{*}\right)\right|_{\mathcal{M}^{(\mathcal{N})}}=\left(\frac{1}{T},-\frac{\mu}{T}\right)$, where $T$ is the temperature and $\mu$ the chemical potential.

\section{Illustration: Kinetic theory}

The Legendre transformation $S^{*}$ in $N$ implied by the fundamental thermodynamic relation described in Step 2 above is $S^{*}\left(n^{*}, e^{*}\right)=-k_{B}\left(\frac{2 \pi m k_{B}}{h^{2} E^{*}}\right)^{3 / 2} e^{-\frac{n^{*}}{k_{B}}}$, which, then, by making another Legendre transformation in $N$, becomes:

$$
S(e, n)=n k_{B}\left(\frac{5}{2}+\ln \left(\frac{1}{n}\left(\frac{4 \pi m e}{3 n h^{2}}\right)^{3 / 2}\right)\right) .
$$

Before leaving equilibrium thermodynamics, we make a comment about a possible path that can be taken to explore further its geometrical content.

The properties of macroscopic systems seen in thermodynamic measurements are expressed in equilibrium thermodynamics in the fundamental thermodynamic relation (13) and (14). This relation has been represented in the geometrical formulation as the manifold $\mathcal{M}^{(\mathcal{N})}$. We are thus in a position to relate directly the experimentally-observed thermodynamic properties to geometrical properties of the manifold $\mathcal{M}^{(\mathcal{N})}$. What kind of geometrical properties can we consider? It is natural to begin with the Riemannian geometry. If we equip the space $\mathbb{M}^{(\mathbb{N})}$ with a metric structure, then the embedding (17) defining $\mathcal{M}^{(\mathcal{N})}$ induces a Riemannian structure on $\mathcal{M}^{(\mathcal{N})}$. We can then explore a possible correspondence between physical properties and geometrical concepts arising in Riemannian geometry. For example, the scalar curvature could be a quantity that can serve as an appropriate geometrical characterization of the behaviour observed in the vicinity of phase transitions and critical points.

This has been indeed suggested by George Ruppeiner and his collaborators in [40]. The investigation reported there differs, however, from the suggestion made in the previous paragraph on two points. The Riemannian geometry is investigated on the level of the classical equilibrium thermodynamics only, and it is introduced on the basis of arguments that are somewhat different from those put forward in the previous paragraph. While the latter difference is less significant, the former is important. If we limit ourselves to the level of the classical equilibrium thermodynamics, then the only way we can get the fundamental thermodynamic relation that corresponds to a given macroscopic system is by making experimental measurements. Indeed, the scalar curvature in the vicinity of critical points is calculated in [41] from the results of experimental observations.

It would be, of course, very interesting and highly desirable to associate physical properties entering mesoscopic levels with the Riemannian geometrical properties seen on the level of the classical equilibrium thermodynamics. The authors of $[40,41]$ use the Einstein theory of fluctuations to reach from the classical equilibrium thermodynamics to mesoscopic levels that involve fluctuations. Alternatively, if we would start with the Riemannian geometry on a mesoscopic level (i.e., with the Riemannian geometry of $\mathcal{M}^{(\mathcal{N})}$ ), we could then, via MaxEnt, arrive at the Riemannian geometry on the level of the classical equilibrium thermodynamics and obtain in this way the desired mesoscopic interpretation of singular geometrical features seen on the level of the classical equilibrium thermodynamics.

We shall not pursue this route here. We shall only comment about critical points and about their emergence in microscopic and mesoscopic theories. It is well known that in the setting of the Gibbs 
equilibrium statistical mechanics of a macroscopic system composed of a finite number of particles, phase transitions and critical points do not arise as singularities, as they do at the level of the classical equilibrium thermodynamics. In other words, phase transitions and critical points remain "invisible" in the Gibbs statistical mechanics with a finite number of particles. How can we make them visible? It is well known that this can be done by the so-called thermodynamic limit in which the Gibbs equilibrium statistical mechanics is lifted to a larger mathematical setting (e.g., the setting of $C^{*}$ algebras; see [42]). There is, however, also another way, suggested (somewhat implicitly) by Melville Green in [43]. Instead of lifting the Gibbs theory to a larger mathematical setting with an infinite number of particles, we go in the opposite direction. We make reductions to formulations involving distribution functions of a smaller number of particles. We then anticipate (see [43]) to be able to see phase transitions and critical phenomena in the emerging complexities of the geometry (in our formulation, it would be the geometry of $\mathcal{M}^{(\mathcal{N})}$ ). How do we do the reductions? From the physical point of view, a reduction is a passage in which we ignore insignificant details and concentrate only on essential features. We anticipate that the ignorance of insignificant details will make the significant features (for instance, those related to phase transitions and critical phenomena) more visible. However, how do we know which details are insignificant and which are not? In principle, the answer lies in the microscopic time evolution. We therefore proceed to investigate it.

\subsection{Geometry of Dynamics}

Most macroscopic systems that are left free from external influences are seen in mesoscopic and macroscopic experimental observations to evolve in such a way that after some time, they reach states (called equilibrium states) in which the observed behaviour is found to be well described by the classical equilibrium thermodynamics. As we have seen in the previous section, the ignored details (in both experimental observations and the mathematical formulation) make the equilibrium states be those at which the entropy reaches its maximum allowed by constraints. We now put forward two additional assumptions. First, we assume that during the time evolution, the entropy does not decrease, and second, that the time evolution is a sequence of infinitesimal Legendre transformations (more precisely, contact structure-preserving transformations). In other words, we assume that the entropy increases monotonically and that the single Legendre transformation maximizing the entropy (described in the previous section) is a infinite sequence of infinitesimal Legendre transformations expressing mathematically the experimentally-observed time evolution.

The next development of the mathematical formulation of the dynamics is straightforward. We keep the setting introduced in the previous section and ask two questions. First, what is the canonical form of the infinitesimal contact structure-preserving transformations, and second, what specifically are such transformations that on the Gibbs-Legendre manifold become those generated by the GENERIC Equation (1). The answer to the first question is well known from contact geometry (see, e.g., [33]): Since we have already appreciated the usefulness of the geometrical formulation offered by the space $\mathbb{M}^{(\mathbb{N})}$, we shall look for the time evolution that takes place in this space and preserves its geometry. We 
begin by recalling (see, e.g., [33]) the canonical form of the time evolution preserving the one-form $\vartheta$ (see (15)).

$$
\begin{aligned}
\dot{x} & =\Psi_{x^{*}} \\
\dot{x}^{*} & =-\Psi_{x}+x^{*} \Psi_{\phi} \\
\dot{y}^{*} & =\Psi_{y} \\
\dot{y} & =-\Psi_{y^{*}}+y \Psi_{\phi} \\
\dot{\phi} & =-\Psi+<x^{*}, \Psi_{x^{*}}>+<y, \Psi_{y}>
\end{aligned}
$$

where $\Psi: \mathbb{M}^{(\mathbb{N})} \rightarrow \mathbb{R}$ is called a contact Hamiltonian.

To answer the second question, we look for $\Psi$ for which the Gibbs-Legendre manifold $\mathcal{M}^{(\mathcal{N})}$ is an invariant manifold, and (18) on this manifold becomes the GENERIC Equation (1). From the contact geometry, we know (see, e.g., [33]) that $[\Psi]_{\mathcal{M}^{(\mathcal{N})}}=0$. However, this requirement, together with the requirement $[(18)]_{\mathcal{M}^{(\mathcal{N})}}=(1)$, still does not single out the contact Hamiltonian $\Psi$. The differences will show in the vector field outside the GL manifold $\mathcal{M}^{(\mathcal{N})}$.

We suggest (see [44]):

$$
\Psi\left(x, x^{*}, y^{*}\right)=-\chi \mathcal{S}\left(x, x^{*}, y^{*}\right)+\frac{1}{e^{*}} \mathcal{H}\left(x, x^{*}, y^{*}\right)
$$

where:

$$
\begin{aligned}
\mathcal{S}\left(x, x^{*}, y^{*}\right) & =\Xi\left(x, x^{*}, y^{*}\right)-\left[\Xi\left(x, x^{*}, y^{*}\right)\right]_{x^{*}=\Phi_{x}} \\
\mathcal{H}\left(x, x^{*}, y^{*}\right) & \left.=<x^{*}, L \Phi_{x}\right\rangle
\end{aligned}
$$

and $\chi: \mathbb{M}^{(\mathbb{N})} \rightarrow \mathbb{R}^{+}$. It is a matter of direct verification to show that (18) with the contact Hamiltonian $\Psi$ given in (19) becomes on the GL manifold $\mathcal{M}^{(\mathcal{N})}$ the GENERIC equation (1).

Illustration: Geometrical formulation of (2).

We take the initial level to be the level on which $x=(p, q, e)$ serve as state variables and the target level with $y=E$ as state variables. The fundamental thermodynamic relation on the initial level (13) is $S=S(p, q, e)$, and (14) is $E=e$. This means that (see (16)) $\Phi\left(p, q, e, E^{*}\right)=$ $-S(p, q, e)+E^{*} e$. The fundamental thermodynamics relation (13) on the target level implied by the fundamental thermodynamic relation on the initial level is $S^{*}=S^{*}\left(E^{*}\right)=\Phi\left((p, q, e)_{e q}\left(E^{*}\right)\right.$, $\left.E^{*}\right)$, where $(p, q, e)_{e q}\left(E^{*}\right)$ is a solution to $\Phi_{p}=0, \Phi_{q}=0, \Phi_{e}=0$. The Gibbs-Legendre manifold $\mathcal{M}^{(\mathcal{N})}$ (see (17)) is now $\left(p, q, e, E^{*}\right) \hookrightarrow\left(p, q, e, \Phi_{p}\left(p, q, e, E^{*}\right), \Phi_{q}\left(p, q, e, E^{*}\right), \Phi_{e}\left(p, q, e, E^{*}\right), E^{*}, e, \Phi\left(p, q, e, E^{*}\right)\right)$.

Now, we turn to the time evolution (2). For the sake of simplicity, we take $\Xi\left(p^{*}\right)=\Lambda\left(p^{*}\right)^{2}$, where $\Lambda>0$ is a parameter. Equation (2) thus becomes:

$$
\left(\begin{array}{c}
\dot{p} \\
\dot{q} \\
\dot{e}
\end{array}\right)=\left(\begin{array}{c}
\frac{S_{q}}{S_{e}}+2 \Lambda S_{p} \\
-\frac{S_{p}}{S_{e}} \\
0
\end{array}\right)
$$

which implies $\dot{\phi}=-2 \Lambda\left(S_{p}\right)^{2}$. In the contact-geometry formulation, we have $\mathcal{H}=\frac{E^{*}}{S_{e}}\left(p^{*} S_{q}-q^{*} S_{p}\right)$; $\mathcal{S}=\Lambda\left(\left(p^{*}\right)^{2}-\left(S_{p}\right)^{2}\right)$, and thus, (see (19)) $\Psi=\Lambda\left(\left(S_{p}\right)^{2}-\left(p^{*}\right)^{2}\right)+\frac{1}{S_{e}}\left(p^{*} S_{q}-q^{*} S_{p}\right)$. This then implies $\Psi_{p^{*}}=-2 \Lambda p^{*}+\frac{1}{S_{e}} S_{q} ; \Psi_{q^{*}}=-\frac{1}{S_{e}} S_{p} ; \Psi_{e^{*}}=0 ; \Psi_{E^{*}}=0 ; \Psi_{E}=0$. From (18), we have: 


$$
\begin{gathered}
\left(\begin{array}{c}
\dot{p} \\
\dot{q} \\
\dot{e} \\
\dot{\phi}
\end{array}\right)=\left(\begin{array}{c}
\frac{S_{q}}{S_{e}}-2 \Lambda p^{*} \\
-\frac{S_{p}}{S_{e}} \\
0 \\
\Psi+\frac{1}{S_{e}}\left(p^{*} S_{q}-q^{*} S_{p}\right)-2 \Lambda\left(p^{*}\right)^{2}
\end{array}\right) \\
{\left[\left(\begin{array}{c}
\dot{p}^{*} \\
\dot{q}^{*} \\
\dot{e}^{*}
\end{array}\right)\right]_{\mathcal{M}^{(\mathcal{N})}}=\left(\begin{array}{lll}
\Phi_{p p} & \Phi_{p q} & \Phi_{p e} \\
\Phi_{p q} & \Phi_{q q} & \Phi_{q e} \\
\Phi_{p e} & \Phi_{e q} & \Phi_{e e}
\end{array}\right)\left[\left(\begin{array}{c}
\dot{p} \\
\dot{q} \\
\dot{e}
\end{array}\right)\right]_{\mathcal{M}^{(\mathcal{N})}}}
\end{gathered}
$$

and $\dot{E}=0, \dot{E}^{*}=0$.

\subsubsection{Alternative Geometrical Formulation of Hamilton's Mechanics}

The principal reason for making the lift from the space $M$ to the space $\mathbb{M}^{(\mathbb{N})}$ was to get a suitable setting for combining symplectic and gradient dynamics. We can now ask the question: What could be a possible advantage of such a lift in the particular case when only symplectic dynamics is present? To answer it, we note that the original geometrical (symplectic) structure in $M$ does not enter in the lift the new geometrical (contact) structure in $\mathbb{M}^{(\mathbb{N})}$, but it enters the new generating potential $\mathcal{H}$ (see (21)) in $\mathbb{M}^{(\mathbb{N})}$. This new view of the symplectic structure with which $M$ is equipped becomes particularly useful if the structure is degenerate. In such a case, the degeneracy (i.e., Casimirs) manifests itself in the landscape of the generating potential $\mathcal{H}$. We note also that the Jacobi identity becomes in $\mathbb{M}^{(\mathbb{N})}$ an integrability condition for the equation $\mathcal{H}=0$.

\subsubsection{Externally-Driven Macroscopic Systems, Complementary Dynamics}

The time evolution governed by (18) brings the starting level (on which $M$ serves as the state space) to the target level that uses $N$ as its state space. We now discuss possible target levels.

The first and the most obvious candidate is the level of the classical equilibrium thermodynamics. In this case, the potential $W$ (see (5)) is the number of moles $N_{\text {mol }}: M \rightarrow \mathbb{R}$. Indeed, the number of moles remains unchanged in both mechanic and thermodynamic evolutions describing the approach to thermodynamic equilibrium states. From the physical point of view, (1), or alternatively, (18), describes in this case the time evolution of externally unforced macroscopic systems that reach thermodynamic equilibrium states at which their behaviour is well described by the classical equilibrium thermodynamics. The advantage of (18) over (1) is that the thermodynamics is manifestly present already in dynamics.

Now, we turn to externally-driven macroscopic systems. In other words, we turn our attention to macroscopic systems that are open towards their environment. For example, we may think of the Rayleigh-Bénard system (a thin horizontal layer of a fluid heated from below). This system is prevented by external forces (the force of gravitation and the imposed temperature gradient) to reach thermodynamic equilibrium states. Nevertheless, the experience shows that the time evolution of the Rayleigh-Bénard system is found to be well described by fluid mechanics equations (in particular, Boussinesq equations). This means that if we take any starting level that involves more details than the level of fluid mechanics, then the time evolution observed on it will show an approach to the time 
evolution taking place on the level of fluid mechanics. It is then this approach that is described by (1) or alternatively (18). The potential $W$ will in this case be $W: M \rightarrow N_{f m}$, where $M$ is the state space used on the initial level and $N_{f m}$ is the state space of fluid mechanics.

In general, we introduce the concept of a complementary dynamics and conjecture that (1), or alternatively (18), governs its time evolution. To define the concept, we note that any well-established mesoscopic dynamics (e.g., fluid mechanics or the Boltzmann kinetic theory) has its complementary dynamics that is defined as dynamics describing the reduction: Microscopic dynamics $\rightarrow$ Mesoscopic dynamics. The Microscopic dynamics is the complete microscopic dynamics (i.e., dynamics of the particles composing the macroscopic system under consideration). We shall also introduce the concept of a partial complementary dynamics as dynamics describing reduction Mesoscopic dynamics $\rightarrow$ Mesoscopic dynamics, where mesoscopic dynamics involves more details than the mesoscopic dynamics. In the particular case when we choose the target level (i.e., the mesoscopic dynamics) to be the level of the classical equilibrium thermodynamics (this means that there is no time evolution taking place on this level), then the complementary dynamics is the complete dynamics. We shall make an additional comment about a general complementary dynamics in the final comment of Section 4.3.

\subsection{Comments}

We recapitulate the gains that we have made by formulating (1) in the form (18), (19) and make some additional comments.

\subsubsection{Unified Formulation of Dynamics and Thermodynamics}

The thermodynamic content of (1) becomes manifestly visible in (18), (19). This, from the physical point of view, amounts to a proof that certain qualitative properties of solutions of (1) agree with the results of experimental observations (namely, the observations showing the multiscale nature of macroscopic physics, like, for example, observations of the approach to states at which mesoscopic theories formulated originally on other levels, e.g., the classical equilibrium thermodynamics, apply).

\subsubsection{A Single Geometrical Structure and Two Generating Potentials}

Two geometrical structures and one generating potential are used to construct the vector field (1). On the contrary, the vector field (18), (19) is constructed with one geometrical structure and two generating potentials.

We recall that the two geometrical structures involved in (1) are the symplectic structure (represented in (1) by the bivector $L$ ) and a generalized Riemannian structure (represented in (1) by the dissipation potential $\Xi$ ). The single generating potential is the thermodynamic potential $\Phi$ (see (16)). We note that due to the degeneracy of the two geometrical structures in (1), we can indeed replace the two potentials appearing in (1), namely the energy $E$ and the entropy $S$, with a single potential $\Phi$ (that is, their linear combination), provided we appropriately modify the multiplicative factor $\chi$.

In (18) and (19), there is only one universal and nondegenerate geometrical structure (the contact structure). This structure is also preserved during the time evolution. All of the particularities of the 
symplectic and Riemannian structures appearing in (1), as well as the fundamental thermodynamic relation (manifesting itself in the Gibbs-Legendre manifold $\mathcal{M}^{(\mathcal{N})}$ ) and generating potentials enter two new generating potentials. One, $\mathcal{H}$, has the physical interpretation of the rate of energy and the other, $\mathcal{S}$, the rate of entropy. (We note that in the context of (1), we cannot talk about the rate of energy, since the energy is conserved. In the context of (18) and (19), the energy is still conserved on $\mathcal{M}^{(\mathcal{N})}$, and thus, $\mathcal{H}_{\mathcal{M}^{(\mathcal{N})}}=0$; however, its gradients, which generate the time evolution, are different from zero).

\subsubsection{Variational Formulation}

It is well known that Hamilton's dynamics can be formulated as a variational problem (Hamilton's variational principle). The variational formulation does not however extend to a dynamics in which Hamilton's dynamics is combined with gradient dynamics (as in (1)). It is therefore interesting to note that (1) in the form (18), (19) does admit the variational formulation. Moreover, the variational formulation has a very clear physical interpretation, namely that the total entropy generated during the time evolution reaches its extremum. We now derive the variational formulation of (18), (19).

We introduce a functional:

$$
\mathcal{I}=\int d t\left[\Psi\left(x, x^{*}, y^{*}\right)-<x^{*}, \dot{x}>\right]
$$

and make the following two observations:

(i) $[\mathcal{I}]_{\mathcal{M}^{(\mathcal{N})}}$ has the physical interpretation of the entropy generated during the time evolution.

(ii) The Euler-Lagrange equations $\delta \mathcal{I}=0$ become on $\mathcal{M}^{(\mathcal{N})}$ equivalent to (1).

\subsubsection{Legendre Transformations: Numerical Approach}

To bring all of the calculations that are needed to make Legendre transformations (as they are presented in Section 4.1) to the end is, in general, a formidable task. This remains to be true also if we accept the assistance of computers and attempt to make the calculations numerically. The dynamic version of Legendre transformations presented in Section 4.2 (as an infinite sequence of infinitesimal contact structure-preserving transformations) may appear to be more convenient from the numerical point of view. Taking the viewpoint presented in Section 4.2, the problem of making Legendre transformations becomes the problem of solving the time evolution governed by (18) on the invariant manifold $\mathcal{M}^{(\mathcal{N})}$. We now suggest to solve (18) numerically. This, of course, is also a difficult problem, but it may be simpler than the problem of making Legendre transformations by translating (by making an appropriate discretization) the direct approach presented in Section 4.1 into a numerical approach.

Here, we make only one comment indicating the possible feasibility of the numerical approach inspired by Section 4.2. We have proven that $\mathcal{M}^{(\mathcal{N})}$ is an invariant manifold of the time evolution governed by (18). For numerical calculations, we need, however, more. We need to know that $\mathcal{M}^{(\mathcal{N})}$ is an attracting manifold (i.e., points in $\mathbb{M}^{(\mathbb{N})}$ that lie outside of $\mathcal{M}^{(\mathcal{N})}$ are approaching $\mathcal{M}^{(\mathcal{N})}$ as $t \rightarrow \infty$ ). This brings us to a question that we have not addressed so far, namely to the the physical meaning and mathematical properties of the time evolution governed by (18) outside the invariant manifold $\mathcal{M}^{(\mathcal{N})}$.

At this point, we only note that we can make many variations in (18), (19) that change the time evolution outside of $\mathcal{M}^{(\mathcal{N})}$, but leave the time evolution on $\mathcal{M}^{(\mathcal{N})}$ unchanged. We can make such 
variations by changing the multiplicative factor $\chi$ in (19) and, more importantly, also by introducing into (18) a "dissipation", similarly as we have introduced a dissipation in (1). One such possible modification is described below.

We limit ourselves only to $\chi$, which depends on $\left(x, x^{*}, y^{*}, y\right)$, introduce a new potential $\Upsilon=\Psi^{2}$ and modify (18) into:

$$
\begin{aligned}
\dot{x} & =\Psi_{x^{*}}-\nu \Upsilon_{x} \\
\dot{x}^{*} & =-\Psi_{x}-\nu \Upsilon_{x^{*}} \\
\dot{y}^{*} & =\Psi_{y}-\nu \Upsilon_{y^{*}} \\
\dot{y} & =-\Psi_{y^{*}}-\nu \Upsilon_{y} \\
\dot{\phi} & =-\Psi+<x^{*}, \Psi_{x^{*}}>+<y, \Psi_{y}>
\end{aligned}
$$

It is now easy to verify that: (i) the time evolution governed by (23) does not preserve any longer the one-form (15); (ii) (18) and (23) coincide on $\mathcal{M}^{(\mathcal{N})}$; (iii) $\frac{d \Upsilon}{d t}=-\nu\left(\left(\Upsilon_{x}\right)^{2}+\left(\Upsilon_{x^{*}}\right)^{2}+\left(\Upsilon_{y^{*}}\right)^{2}+\left(\Upsilon_{y}\right)^{2}\right) \leq 0$, provided the coefficient $\nu>0$; and (iv) $\Upsilon \geq 0$ and $[\Upsilon]_{\mathcal{M}^{(\mathcal{N})}}=0$. If, moreover, we were able to prove that $\Upsilon$ is convex in a neighbourhood of $\mathcal{M}^{(\mathcal{N})}$, then the modification (23) of (18) would indeed be suitable for numerical calculations of Legendre transformations.

\subsubsection{Entropy}

Let us choose a mesoscopic dynamical theory (representing the target level that we denote Level " $\mathrm{t}$ ") and consider it together with its complement (representing the initial level, denoted Level "c"). We recall (see the last comment in Section 4.2) that the complementary dynamics is the dynamics describing the approach Level "c" $\rightarrow$ Level " $t$ ". In the case when Level "c" is not the most microscopic level on which all details are taken into account, then the dynamics is called a partial complementary dynamics. We now discuss several choices of target and complementary levels and discuss the entropies that arise.

Example 1. We choose the initial Level " $c$ " to be the the level on which all details are taken into account, and Level " $t$ " is absent. Level " $c$ " is the level on which the macroscopic system is seen as being composed of particles whose time evolution is governed by the classical particle dynamics. In this case, there is no entropy in the mathematical description of the time evolution, since no details are ignored.

Example 2. We choose Level " $t$ " to be the level of the classical equilibrium thermodynamics, and, as its complement, we keep Level "c" from Example 1 above. In this setting, there are two entropies: one denoted $S^{(c \rightarrow t)}$ driving the approach Level " $c$ " $\rightarrow$ Level " $t$ " and $S^{(t \leftarrow c)}$, that is the entropy on the target Level " $t$ " obtained by evaluating $S^{(c \rightarrow t)}$ at the states that are reached (as $t \rightarrow \infty$ ) in the time evolution Level "c" $\rightarrow$ Level " $t$ ". It is the entropy $S^{(t \leftarrow c)}$ that is the entropy of the classical equilibrium thermodynamics, where it is however introduced completely without any reference to Level "c". We see now that its origin lies in the potential generating approach to thermodynamic equilibrium states. If we restrict ourselves only to Level " $t$ " (i.e., to the classical equilibrium thermodynamics in this case), then the association between macroscopic systems and the entropies (called a fundamental 
thermodynamic relation) can be obtained only by making experimental measurement (collected in the so-called thermodynamic tables). If the Level " $c$ " is chosen to be the level of kinetic theory, then the equation governing the time evolution Level " $c$ " $\rightarrow$ Level " $t$ " is a particular representation of (1) corresponding to the Boltzmann kinetic equation (with $S^{(c \rightarrow t)}$ being the Boltzmann entropy; see Section 4.1). The resulting entropy $S^{(t \leftarrow c)}$ on the level of the classical equilibrium thermodynamics is the entropy characterizing ideal gas. If we choose Level "c" to the most microscopic level (i.e., the level on which macroscopic systems are seen as composed of microscopic particles), then it is rather difficult to identify a particular realization of (1) governing the approach Level " $c$ " $\rightarrow$ Level " $t$ ". We can however follow the Gibbs equilibrium statistical mechanics and replace (1) with the requirement of the conservation of mass and energy and with the ergodicity-type hypothesis about particle trajectories. We choose then $S^{(c \rightarrow t)}$ to be the Gibbs entropy. The MaxEnt principle (see Section 4.1) brings us to the entropy $S^{(t \leftarrow c)}$. In the particular case when we fix the energy, the number of particles and the volume (the so-called microcanonical ensemble), then we can interpret physically $S^{(t \leftarrow c)}$ that arises in the MaxEnt as a quantity that is proportional to the logarithm of the volume of the region in the microscopic state space (i.e., the state space used on Level "c") that corresponds to a single macroscopic state (i.e., a point in the state space used on Level " $t$ ").

Example 3. In this example, we choose the target Level " $t$ " to be a mesoscopic level on which time evolution takes place (e.g., the level of fluid mechanics). Here, we make two important observations.

(i) We note that irrespective of the nature of the time evolution taking place on Level " $t$ " (it may be, in the case of externally unforced macroscopic systems, the time evolution governed by a particular realization of (1) describing the approach to the thermodynamic equilibrium states, or, in the case of externally-driven macroscopic systems, it may be a completely general time evolution), the approach Level " $c$ " $\rightarrow$ Level " $t$ " is generated by (1) with the entropy $S^{(c \rightarrow t)}$. Irrespective of the nature of the time evolution taking place on Level " $t$ ", we thus obtain an entropy $S^{(t \leftarrow c)}$ on the target Level " $t$ ".

(ii) The states approached in the time evolution representing the reduction Level " $c$ " $\rightarrow$ Level " $t$ " represent a submanifold (we denote it by the symbol $\mathcal{M}^{(c \rightarrow t)}$ ) on which the time evolution on Level " $c$ " becomes the time evolution on Level " $t$ ". The manifold $\mathcal{M}^{(c \rightarrow t)}$ is also called a closure manifold. Since $\mathcal{M}^{(c \rightarrow t)}$ is also the manifold on which $S^{(c \rightarrow t)}$ reaches its maximum, the entropy $S^{(t \leftarrow c)}=\left[S^{(c \rightarrow t)}\right]_{\mathcal{M}^{(c \rightarrow t)}}$ contains information about the manifold $\mathcal{M}^{(c \rightarrow t)}$ that is different, but consistent with and complementary to the information provided by the time evolution taking place on Level " $t$ ".

Summing up, we emphasize that the fact that there exists an autonomous Level " $t$ " (its existence is established by making a comparison with the results of experimental observations) implies that the complementary dynamics approaches Level " $\mathrm{t}$ ", and the entropy generating such an approach appears on Level "t" as entropy $S^{(t \leftarrow c)}$. If Level " $\mathrm{t}$ " is the level of the classical equilibrium thermodynamics on which no time evolution takes place, then $S^{(t \leftarrow c)}$ obtained in this way is the entropy of the classical equilibrium thermodynamics. If, on the other hand, Level " $t$ " is a mesoscopic level on which time evolution takes place (it can be the time evolution of an externally-driven macroscopic system), then $S^{(t \leftarrow c)}$ is an entropy providing alternative and complementary information about the time evolution on Level " $\mathrm{t}$ ". 


\subsubsection{Alternative Geometrical Formulations of Nonequilibrium Thermodynamics}

The geometrical formulation of nonequilibrium thermodynamics presented above was motivated by the geometrical formulation of equilibrium thermodynamics. Other attempts, with different motivations, have been made to extend the geometrical formulation of classical mechanics to a mesoscopic dissipative mechanics. We mention some of them below.

Darryl Holm has carried his elegant geometrical formulation of non-dissipative fluid mechanics [45] to certain types of dissipative fluid mechanics by introducing what he calls Casimir dissipation [46]. The time evolution equations are modified in such a way that energy remains to be conserved, but one of the Casimirs is allowed to dissipate. However, this is exactly the entropic dissipation, since entropy is, as we have argued in this paper, the physically (thermodynamically)-appropriate Casimir to dissipate. We suggest that the contact structure formulation (of the type developed in this paper) is then a natural next step to continue the line of arguments introduced in [46].

A rigorous mathematical investigation of dissipative dynamics (that includes the dynamics arising in chemical kinetics) has led Alexander Mielke $[31,47]$ to various generalizations of gradient dynamics that are not equivalent (though closely related) to the generalizations involving dissipation potentials.

A systematic search for quasi-invariant manifolds, $\mathcal{M}^{(c \rightarrow t)}$, on which a given mesoscopic dynamics (on Level "c") reduces to another mesoscopic dynamics (on Level " $\mathrm{t}$ ") involving less details is presented in [48]. The general method described there is seen as an extension of the Chapman-Enskog method (developed for passing from the Boltzmann kinetic equation to the Navier-Stokes-Fourier fluid mechanics) to a general mesoscopic dynamics. The first step in the method is an introduction of the zero approximation $\mathcal{M}_{0}^{(c \rightarrow t)}$ of $\mathcal{M}^{(c \rightarrow t)}$ (obtained via MaxEnt from an entropy $S_{0}^{(c \rightarrow t)}$ ). In the second step, $\mathcal{M}_{0}^{(c \rightarrow t)}$ is deformed into $\mathcal{M}_{1}^{(c \rightarrow t)}$ in such a way that $\mathcal{M}_{1}^{(c \rightarrow t)}$ is more invariant (i.e., the vector field of the "c"-mesoscopic dynamics is sticking out of the tangent planes of $\mathcal{M}_{1}^{(c \rightarrow t)}$, less than it is sticking out of the tangent planes of $\mathcal{M}_{0}^{(c \rightarrow t)}$ ). Having in mind the thermodynamic viewpoint of dynamics developed in this paper, we then continue and take another step, namely we identify the modification $S_{1}^{(c \rightarrow t)}$ of the entropy $S_{0}^{(c \rightarrow t)}$ that gives, via MaxEnt, the modified manifold $\mathcal{M}_{1}^{(c \rightarrow t)}$.

Problems arising in optimization have led in [49,50] to a geometrical formulation of dissipative dynamics that uses the contact structure geometry and that appears to be closely related to the formulation developed in this paper.

\section{Concluding Remarks}

Equations governing the time evolution in the classical nonequilibrium thermodynamics combine symplectic and gradient dynamics. From the geometrical point of view, their formulation thus involves two geometrical structures (namely, symplectic and Riemannian) that transform gradients of two generating potentials (energy and entropy) into vector fields. Due to degeneracies of the geometric structures (guaranteeing required time evolution of mass, energy and entropy), the two generating potentials can be combined into one. The classical nonequilibrium thermodynamics thus needs two geometrical structures and one generating potential to write down its governing equations. Moreover, in order to express the thermodynamic content of the formulation, the governing equations are supplemented in the classical formulation with the so-called entropy equation. This is an extra equation 
expressing the fundamental thermodynamic relation (i.e., entropy as a function of the state variables) and the requirement that the entropy does not decrease during the time evolution. From the geometrical point of view, the entropy equation (and thus, the thermodynamic content of the formulation) remains outside the geometry.

On the other hand, in the formulation (18), (19), the thermodynamic content becomes manifestly demonstrated. The time evolution is a continuous sequence of infinitesimal Legendre transformations (contact structure-preserving transformations); the time evolution takes place on the Gibbs-Legendre manifold expressing geometrically the fundamental thermodynamic relation, and the symplectic and Riemannian geometry, as well as the generating potentials of the classical formulation, appear in the formulation (18), (19) in two new generating potentials having the physical interpretation of the rate of energy and the rate of entropy.

\section{Acknowledgements}

The research reported in this paper has been partially supported by Natural Sciences and Engineering Research Council of Canada.

\section{Conflicts of Interest}

The author declares no conflict of interest.

\section{References}

1. Clebsch, A. Über die Integration der Hydrodynamische Gleichungen. Journal für die reine und angewandte Mathematik 1895, 56, 1-10.

2. Arnold, V. Sur la géometrie différentielle des groupes de Lie de dimension infini et ses applications dans l'hydrodynamique des fluides parfaits. Annales de l'institut Fourier 1966, 16, 319-361.

3. Marsden, J.; Weinstein, A. Coadjoint orbits, vortices, and clebsch variables for incompressible fluids. Physica D 1983, 7, 305-323.

4. Ginzburg, V.; Landau, L. On the theory of superconductivity. Zhur. Eksp. Theor. Fiz. 1950, 20, 1064-1082.

5. Cahn, J.; Hilliard, J. Free Energy of a Nonuniform System. I. Interfacial Free Energy. J. Chem. Phys. 1958, 28, 258-267.

6. Dzyaloshinskii, I.E.; Volovick, G.E. Poisson brackets in condense matter physics. Ann. Phys. 1980, 125, 67-97.

7. Grmela, M. Particle and Bracket Formulations of Kinetic Equations. Contemp. Math. 1984, 28, 125-132.

8. Morrison, P.J. Bracket formulation for irreversible classical fields. Phys. Lett. A 1984, 100, 423-427.

9. Kaufman, A. Dissipative Hamiltonian systems: A unifying principle. Phys. Lett. A 1984, 100, 419-422.

10. Grmela, M. Bracket formulation of diffusion-convection equations. Physica D 198, 21, 179-212. 
11. Beris, A.; Edwards, B. Thermodynamics of Flowing Systems; Oxford University Press, Oxford, UK, 1994.

12. Grmela, M.; Öttinger, H.C. Dynamics and thermodynamics of complex fluids. I. Development of a general formalism. Phys. Rev. E 1997, 56, 6620-6632.

13. Öttinger, H.C.; Grmela, M. Dynamics and thermodynamics of complex fluids. II. Illustrations of a general formalism. Phys. Rev. E 1997, 56, 6633-6655.

14. Truesdell, C. Rational Thermodynamics, 2nd ed.; Springer: New York, NY USA, 1984.

15. Martinas, K.; Ropolyi, L. Analogies: Aristotelian and Modern Physics. Inter. Studies Philos. Sci. 1987, 2, doi:10.1080/02698598708573299.

16. De Groot, S.R.; Mazur, P. Non-equilibrium Thermodynamics; Dover Publications: New York, NY, USA, 1984.

17. Grmela, M. Reciprocity relations in thermodynamics. Physica A 2002, 309, 304-328.

18. Pavelka, M.; Klika, V.; Grmela, M. Time reversal in nonequilibrium thermodynamics. Phys. Rev. E 2014, 90, doi:10.1103/PhysRevE.90.062131.

19. Marsden, J.; Ratiu, T.; Weinstein, A. Semidirect products and reduction in mechanics. Trans. Am. Math. Soc. 1984, 281, 147-177.

20. Marsden, J.; Ratiu, T.S. Introduction to Mechanics and Symmetry; 2nd ed.; Springer: New York, NY, USA, 1999.

21. Grmela, M. Contact Geometry of Mesoscopic Thermodynamics and Dynamics. Entropy 2014, 16, 1652-1686.

22. Grmela, M. Fluctuations in extended mass-action-law dynamics. Physica D 2012, 241, 976-986.

23. DiPerna, R.; Lions, P. Global solutions of Boltzmann's equation and the entropy inequality. Arch. Rational Mech. Anal. 1991, 114, 47-55.

24. Grad, H. On Boltzmann's H-theorem. J. Soc. Indust. Math. 1965, 13, 259-277.

25. Desvillettes, L.; Villani, C. On the trend to global equilibrium for spatially inhomogeneous kinetic systems: The Boltzmann equation. Invent. Math. 2005, 159, 245-316.

26. Godunov, S. Symmetric form of the magnetohydrodynamic equation. Chislennye Metody Mekhaniki Sploshnoi Sredy 1972, 3, 26-34.

27. Friedrichs, K. Conservation equations and the laws of motion in classical physics. Commun. Pure Appl. Math. 1978, 31, 123-131.

28. Friedrichs, K.; Lax, P. Systems of conservation equations with a convex extension. Proc. Natl. Acad. Sci. USA 1971, 68, 1686-1688.

29. Godunov, S.; Romensky, E. Computational Fluid Dynamics Review; Wiley: New York, NY, USA, 1995; pp. 19-31.

30. Öttinger, H. Beyond Equilibrium Thermodynamics; Wiley: New York, NY, USA, 2005.

31. Mielke, A. A gradient structure for reaction-diffusion systems and for energy-drift-diffusion systems. Nonlinearity 2011, 24, 1329-1346.

32. Beretta, C. Steepest entropy ascent model for far-nonequilibrium thermodynamics: Unified implementation of the maximum entropy production principle. Phys. Rev. E 2014, 90, doi:10.1103/PhysRevE.90.042113.

33. Arnold, V.I. Mathematical Methods of Classical Mechanics; Springer: New York, NY, USA, 1989. 
34. Gibbs, J.W. Collected Works; Longmans Green and Company: New York, NY, USA, 1984.

35. Jaynes, E.T. Foundations of probability theory and statistical mechanics. In Delaware Seminar in the Foundation of Physics; Bunge, M., Ed.; Springer: New York, NY, USA, 1967.

36. Hermann, R. Geometry, Physics and Systems; Marcel Dekker: New York, NY, USA, 1984.

37. Mrugala, R.; Nulton, J.; Schoen, J.; Salamon, P. Statistical approach to the geometric structure of thermodynamics. Phys. Rev. A 1990, 41, 3156-3160.

38. Borgnakke, C.; Sonntag, R.E. Fundamentals of Thermodynamics, 7th ed.; Wiley: Hoboken, NJ, USA, 2009.

39. Callen, H. Thermodynamics: An Introduction to the Physical Theories of Equilibrium Thermostatics and Irreversible Thermodynamics; Wiley: Hoboken, NJ, USA, 1960.

40. Ruppeiner, G. Riemannian geometry in thermodynamic fluctuation theory. Rev. Mod. Phys. 1995, 67, doi:10.1103/RevModPhys.67.605.

41. May, H.O.; Mausbach, P.; Ruppeiner, G. Thermodynamic curvature for attractive and repulsive intermolecular forces. Phys. Rev. E 2013, 88, doi:10.1103/PhysRevE.88.032123.

42. Ruelle, D. Thermodynamic Formalism; Addison-Wesley: Boston, MA, USA, 1978.

43. Green, M. Generalized Ornstein-Zernike Approach to Critical Phenomena. J. Math. Phys. 1968, 9, 875-890.

44. Grmela, M. Thermodynamical Lift of the Nonlinear Onsager-Casimir Vector Field. In Proceedings of the Workshop on Hamiltonian Systems, Transformation Groups and Spectral Transform Methods, Université de Montréal, Montréal, QC, Canada, 20-26 October 1990; pp. 199-207.

45. Holm, D. Geometric mechanics: Part I. Dynamics and Symmetry; Imperial College Press: London, UK, 2011.

46. Gay-Malmaz, F; Holm, D. Selective decay by Casimir dissipation in inviscid fluids. Nonlinearity 2013, 26, 495-524.

47. Mielke, A. Thermomechanical modeling of energy-reaction-diffusion systems, including bulk-interface interactions. Discret. Contin. Dyn. Syst. Ser. S 2013, 6, 479-499.

48. Gorban, A.; Karlin, I. Invariant Manifolds for Physical and Chemical Kinetics; Springer: Berlin/Heidelberg, Germany, 2005.

49. Favache, A.; Dochain, D.; Maschke, B. An entropy-based formulation of irreversible processes based on contact structures. Chem. Eng. Sci. 2010, 65, 5204-5216.

50. Ramirez, H.; Maschke, B.; Sharbaro, D. Irreversible port-Hamiltonian systems: A general formulation of irreversible processes with application to CSTR. Chem. Eng. Sci. 2013, 89, 223-234.

(C) 2015 by the author; licensee MDPI, Basel, Switzerland. This article is an open access article distributed under the terms and conditions of the Creative Commons Attribution license (http://creativecommons.org/licenses/by/4.0/). 